\title{
No room to breathe: the importance of lung hyperinflation in COPD
}

\author{
*Mike Thomas', Marc Decramer², Denis E O'Donnell ${ }^{3}$ \\ Department of Primary Care Research, University of Southampton, Southampton, UK \\ ${ }^{2}$ Respiratory Division, University Hospitals, Leuven, Belgium \\ ${ }^{3}$ Division of Respirology, Queen's University and Kingston General Hospital, Kingston, Ontario, Canada
}

Received 23rd October 2012; revised 19th December 2012; accepted 22nd December 2012; online 21st February 2013

\begin{abstract}
Patients with chronic obstructive pulmonary disease (COPD) are progressively limited in their ability to undertake normal everyday activities by a combination of exertional dyspnoea and peripheral muscle weakness. COPD is characterised by expiratory flow limitation, resulting in air trapping and lung hyperinflation. Hyperinflation increases acutely under conditions such as exercise or exacerbations, with an accompanying sharp increase in the intensity of dyspnoea to distressing and intolerable levels. Air trapping, causing increased lung hyperinflation, can be present even in milder COPD during everyday activities. The resulting activity-related dyspnoea leads to a vicious spiral of activity avoidance, physical deconditioning, and reduced quality of life, and has implications for the early development of comorbidities such as cardiovascular disease. Various strategies exist to reduce hyperinflation, notably long-acting bronchodilator treatment (via reduction in flow limitation and improved lung emptying) and an exercise programme (via decreased respiratory rate, reducing ventilatory demand), or their combination. Optimal bronchodilation can reduce exertional dyspnoea and increase a patient's ability to exercise, and improves the chance of successful outcome of a pulmonary rehabilitation programme. There should be a lower threshold for initiating treatments appropriate to the stage of the disease, such as long-acting bronchodilators and an exercise programme for patients with mild-to-moderate disease who experience persistent dyspnoea.
\end{abstract}

(C) 2013 Primary Care Respiratory Society UK. All rights reserved.

M Thomas et al. Prim Care Respir J 2013; 22(1): 101-111

http://dx.doi.org/10.4104/pcrj.2013.00025

Keywords COPD, dyspnoea, exacerbations, exercise, hyperinflation, inspiratory capacity

The full version of this paper, with online appendices,

is available online at www.thepcrj.org

\section{Introduction}

Worldwide, chronic obstructive pulmonary disease (COPD) is estimated to affect $10 \%$ of the population aged $>40$ years. ${ }^{1}$ In primary care in the UK, the estimated prevalence of COPD is $7 \%$ of those aged $\geq 45$, increasing to $8-9 \%$ of those aged $>65 .{ }^{2}$ However, underdiagnosis is a problem, and the true prevalence is likely to be much higher. Using specific screening for COPD, a study among primary care practices in Canada found a prevalence of $21 \%$ among those aged over $40 .{ }^{3}$ COPD is an important cause of mortality and morbidity, ${ }^{4}$ and even patients in the milder stages of disease are at increased risk. ${ }^{5}$

Many patients with COPD can no longer do the activities they used to do, or cannot sustain them to the same extent, because they are limited by respiratory difficulty or dyspnoea. ${ }^{6-8}$ Activityrelated dyspnoea is among the earliest and most troublesome symptoms of COPD, and it progresses over time to incapacitating levels. ${ }^{9}$ Limitations on activity often lead to feelings of social isolation and psychological problems and reduce a patient's perceived quality of life, which is further diminished by the resulting inactivity and physical deconditioning. ${ }^{6}$ The mechanisms underlying activity limitation and dyspnoea are complex, and the situation is further complicated by the problems of ageing and co-morbidity that are common in patients with COPD.

We performed a review of the current medical literature investigating the mechanisms of hyperinflation and its effects on the dyspnoea and activity limitation experienced by patients with COPD. Various treatment modalities have been employed to overcome the problem, and the effects of pharmacological treatment on hyperinflation are summarised. The summary of

\footnotetext{
* Corresponding author: Professor Mike Thomas, Professor of Primary Care Research, University of Southampton; Aldermoor Health Centre, Aldermoor Close, Southampton SO16 5ST, UK. Tel: +44 (0) 2380241050 Fax: +44 (0) 2380701125 E-mail: D.M.Thomas@soton.ac.uk.
} 
treatment effects was based on a literature search of the PubMed database (no date limits) for COPD and terms relating to hyperinflation, narrowed by individual drug name (see Appendix 1 , available online at www.thepcrj.org, for complete search strategy).

\section{Mechanisms underlying exercise limitation in COPD}

COPD is a highly heterogeneous condition with many different factors contributing to its pathophysiology, and the relative contribution of these factors varies from patient to patient. The term COPD principally encompasses two conditions - chronic bronchitis and emphysema. Chronic bronchitis is characterised by airways obstruction resulting from inflammation and remodelling of the larger airways, with oedema and increased mucus production. ${ }^{10}$ Emphysema is characterised by irreversible damage to the lung parenchyma and adjacent vasculature. A recent study suggests that obliteration and narrowing of terminal bronchioles may precede the development of destructive emphysema. ${ }^{11}$ The loss of lung elastic recoil pressure reduces the driving pressure for flow during expiration: expiratory flow rates are diminished at any given lung volume compared with health. In addition, the loss of alveolar walls and attachments, which normally help to maintain airway patency, renders the airways more liable to collapse during expiration. Collectively, these changes give rise to expiratory flow limitation.

Changes to the elastic properties of the lungs in emphysema alter the balance of forces between the lung (inward recoil) and chest wall (outward recoil), so that the relaxation volume of the respiratory system at the end of quiet expiration (end-expiratory lung volume; EELV) is reset to a higher volume than is predicted in health (i.e. static lung hyperinflation). In the presence of expiratory flow limitation, the ability to empty the lungs with each breath is critically dependent on the time available for expiration.

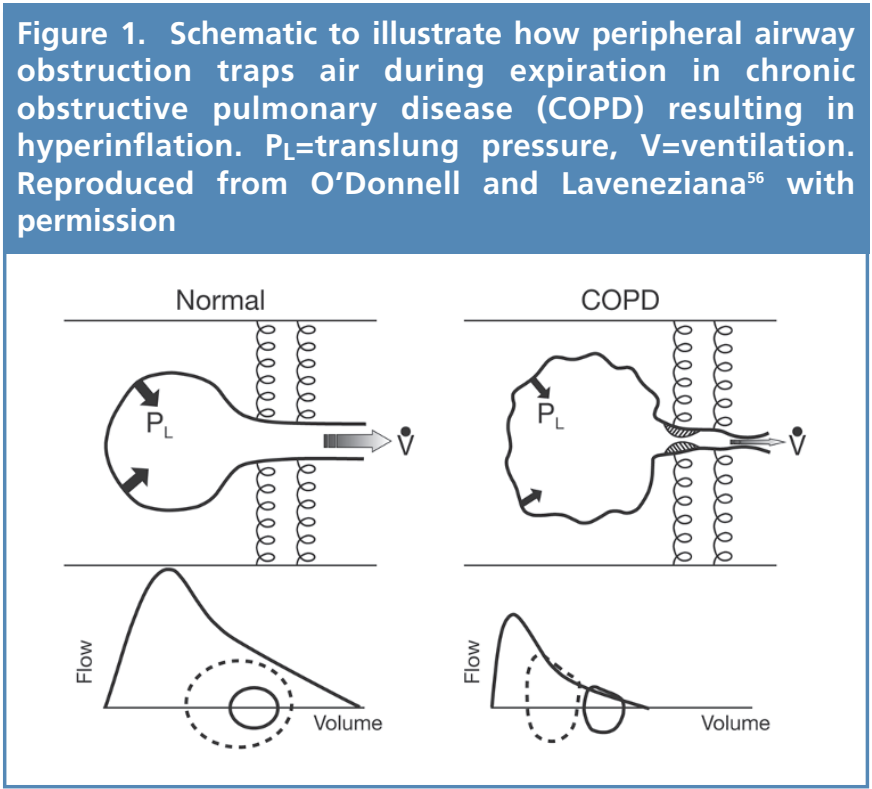

In many patients with flow-limited COPD, expiratory time during spontaneous resting breathing is simply insufficient to allow full lung emptying and gas trapping is the result (Figure 1).

EELV can increase temporarily and to a variable extent above the resting value in situations where expiratory flow limitation is suddenly worsened (i.e. bronchospasm or exacerbation) or when ventilatory demand is abruptly increased, such as with exertion, anxiety, or transient hypoxaemia. ${ }^{12}$ This is termed 'dynamic lung hyperinflation'. In conditions of increased metabolic demand such as exercise, when breathing is accelerated, increased gas trapping becomes inevitable. The presence of lung overinflation limits the ability of tidal volume to expand, and ventilation can only be increased by faster breathing, contributing to further hyperinflation in a vicious cycle.

Although not discussed here, many other factors contribute to limiting a patient's activity in COPD, not least peripheral muscle weakness and deconditioning due to factors such as ageing, poor nutrition, and co-morbidities. ${ }^{13-15}$

\section{Pressure-volume relationship}

From the start of an intake of breath after fully emptying the lungs (residual volume, RV) to the finish (total lung capacity, TLC), the relationship between chest wall pressure and lung volume follows an S-shaped curve. ${ }^{16}$ Healthy subjects breathe at around the midpoint of the curve where volume can increase at comfortable pressure. With their larger lung volume due to gas

Figure 2. Lung volumes at rest and during exercise in healthy subjects and in patients with chronic obstructive pulmonary disease (COPD). In normal lungs, endexpiratory lung volume (EELV) remains relatively constant during exercise as tidal volume can increase and inspiratory capacity (IC) is maintained. Patients with COPD breathe with a greater EELV and less IC. During exercise, as ventilation increases, the increased EELV (dynamic hyperinflation) pushes tidal volume closer to total lung capacity (TLC) where expansion is limited by high pressures. IC decreases and breathing becomes so restricted that patients have to stop activity. $R V=$ residual volume, IRV=inspiratory reserve volume. Reproduced from O'Donnell et al. ${ }^{16}$ with permission
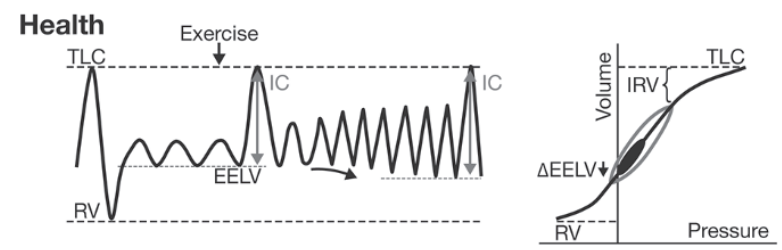

COPD
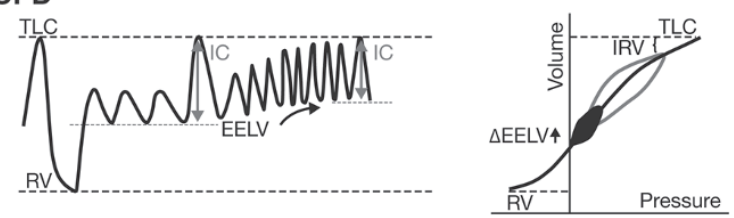
trapping, COPD patients breathe higher up the curve where the breathing muscles have to overcome relatively larger 'elastic' forces to achieve the same increase in lung volume during breathing (Figure 2).

Certain adaptive responses occur to compensate for chronic hyperinflation, including adaptations of the chest wall and diaphragm shape to accommodate the increased volume, and adaptations of muscle fibres to preserve strength and increase endurance in the face of chronic intrinsic mechanical loading. ${ }^{17}$ However, these compensatory mechanisms are quickly overwhelmed when ventilatory demand increases acutely (e.g. during exercise). ${ }^{18-20}$

\section{Exercise}

Because of the already high operating volumes in COPD, any expansion in tidal volume during exercise can be gained only by greater force generation (and contractile effort) by the inspiratory muscles (see Figure 2). Expiratory flow limitation and the accompanying dynamic hyperinflation force the contractile units of the inspiratory muscles to operate at a shorter disadvantageous length, which ultimately weakens the muscles. Inflammation and oxidative stress increase the susceptibility of respiratory muscles to contractile dysfunction under acute inspiratory loading. ${ }^{21}$

\section{Exacerbations of COPD}

An acute increase in hyperinflation can also occur during exacerbations, and is believed to be an important contributor to the characteristic symptom of worsening dyspnoea. ${ }^{12,22,23}$ COPD exacerbations differ greatly in their clinical presentation, reflecting differences in patients' clinical characteristics, the presence of co-morbidities such as chronic heart failure, the underlying pathophysiology of COPD, and causative factors. ${ }^{24}$ Exacerbations involve increased airway inflammation and worsening airway obstruction, in variable contributions. The effectiveness of various non-steroidal treatment modalities in reducing the severity and frequency of exacerbations (bronchodilator drugs, surgery) ${ }^{25,26}$ and the differential effect of bronchodilators and corticosteroids on exacerbations ${ }^{27}$ suggest that exacerbations can involve worsening airway obstruction in the absence of airway inflammation, ${ }^{12}$ assuming that these bronchodilators do not exert anti-inflammatory effects in patients with COPD in vivo.

Studies in hospitalised patients with acute respiratory failure undergoing mechanical ventilation have shown that severe exacerbations involve a mechanism of critical expiratory flow limitation with lung hyperinflation, with serious mechanical consequences. ${ }^{28}$ This can cause fatigue or overt failure of the respiratory muscles. Less is known about the mechanisms behind symptomatic deterioration during mild-to-moderate exacerbations that are commonly encountered in clinical practice, but the underlying physiology is likely to be similar to that of severe exacerbations. ${ }^{12,22}$

\section{Sensory experience of breathlessness in COPD}

In conditions such as exercise and exacerbation, the drive to breathe is increased but the ability of the respiratory system to respond appropriately is greatly hindered. This disparity between the increased central drive to breathe and the mechanical/muscular response of the respiratory system is termed 'neuromechanical uncoupling' or dissociation. Although patients try to meet the increased ventilatory demand, they cannot increase the tidal volume very much (constrained at one end by increased EELV and at the other by the fact that they are forced to breathe close to TLC; Figure 2). With literally no room to breathe despite near-maximal neural drive, patients experience intolerable dyspnoea very quickly.

While COPD patients - in common with healthy subjects describe their dyspnoea on exercise in terms of increased effort and heaviness of breathing, they also describe some unique sensations such as "can't get enough air in" or "unsatisfied inspiratory effort" (Figure 3). ${ }^{29}$ This is somewhat akin to 'air hunger' which can be induced experimentally in healthy volunteers when $\mathrm{CO} 2$ loading is combined with mechanical restriction of lung volume expansion. ${ }^{30}$ This suggests that COPD patients are receiving abnormal peripheral neurosensory information from various mechanical receptors in the respiratory muscles and chest wall, signalling that respiration is inadequate for the excessive effort expended. The perception of unsatisfied effort is rarely reported in healthy subjects.

The change in description of the sensation from 'work and effort' to one of 'unsatisfied inspiration' occurs when the drive to

Figure 3. Qualitative descriptors of exertional dyspnoea (at the end of symptom-limited cycle exercise) of 'unsatisfied inspiration', 'inspiratory difficulty', and 'shallow breathing' were more common among patients with chronic obstructive pulmonary disease (COPD) than in healthy subjects $\left({ }^{*} p<0.05\right)$. Modified from $O^{\prime}$ Donnell et al. ${ }^{29}$ with permission

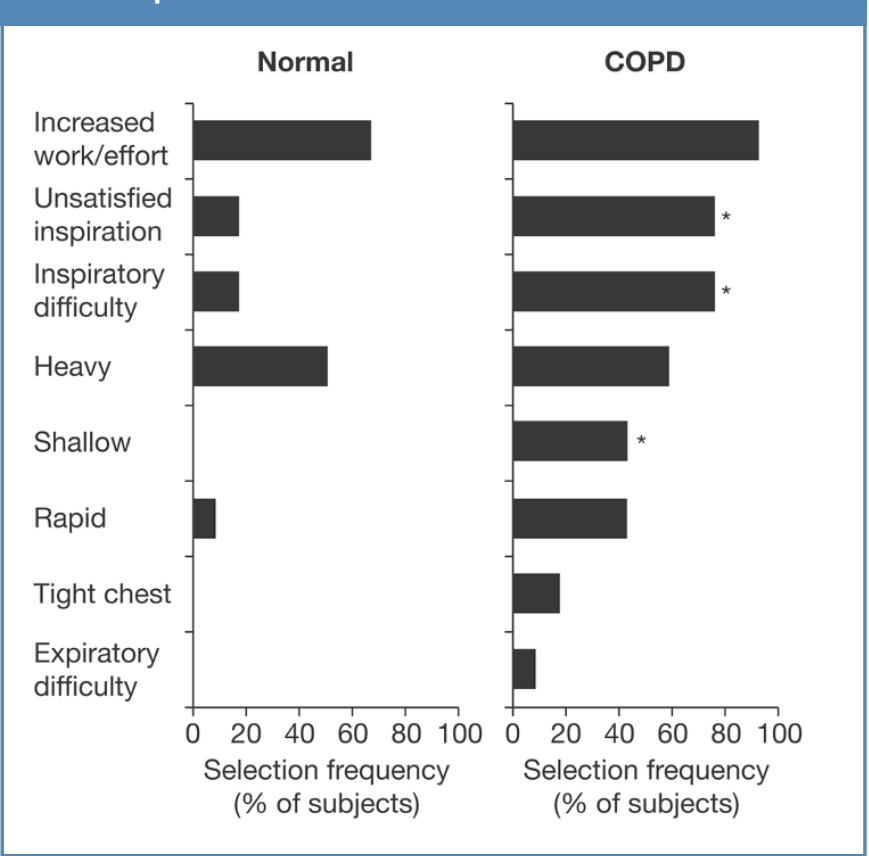


Figure 4. In a study in hyperinflated subjects with chronic obstructive pulmonary disease (COPD), with increasing breathing frequency, tidal volume (VT) expanded to a maximal value of approximately $75 \%$ of the concurrent inspiratory capacity (IC) (left). When this plateau in VT/IC occurs, and regardless of baseline differences in lung function, dyspnoea rises sharply to intolerable levels (right). Q=quartile of COPD severity (airflow limitation). Forced expiratory volume in 1 second quartiles were mean $62 \%, 49 \%, 39 \%$, and $27 \%$ predicted. Modified from O'Donnell et al.32 with permission)
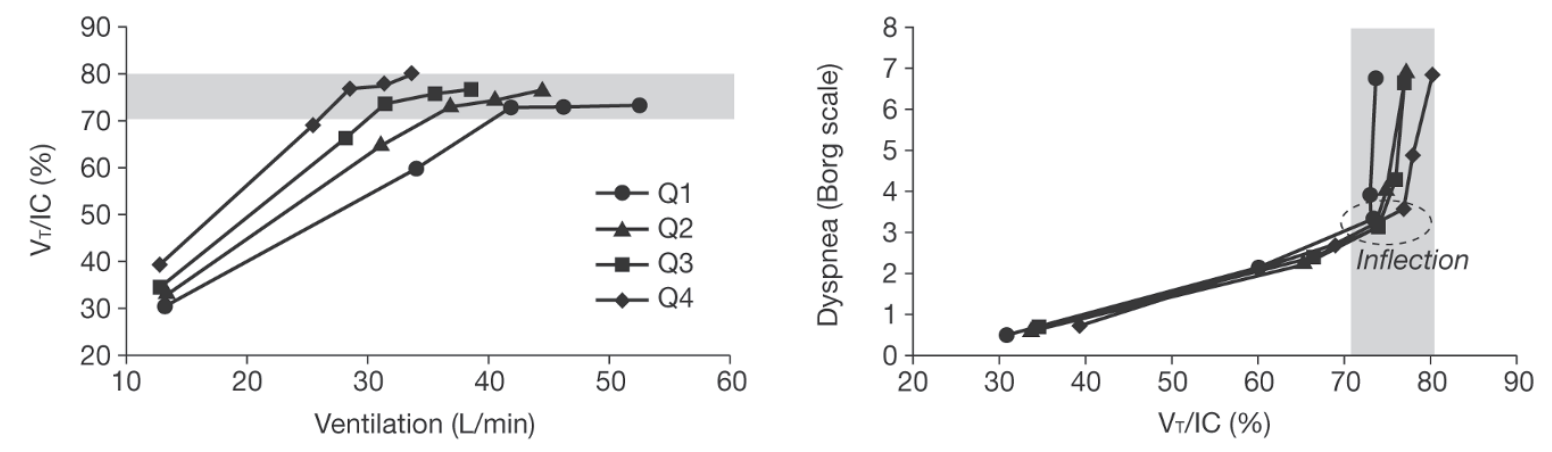

breathe continues to increase without a corresponding increase in lung volume, and the intensity of dyspnoea rises sharply. This inflection point in the relationship between volume and effort during exercise, when tidal volume can no longer increase in line with increasing ventilatory drive, is depicted in Figure 4..$^{31,32}$ The relationship between the increase in dyspnoea and the point where tidal volume is close to TLC is similar across differing levels of baseline airway obstruction. ${ }^{32}$

The perception of unsatisfied inspiration is likely to evoke a powerful fear response that escalates to panic, or respiratory distress. ${ }^{29}$ In general, COPD subjects are distinct from healthy subjects in describing their sensation of breathlessness in terms such as 'frightening', 'helpless', and 'awful'. ${ }^{33}$ The descriptions of dyspnoea that reflect fear and anxiety become more common with increasing severity of COPD. ${ }^{34}$ This powerful affective dimension of dyspnoea has been highlighted in a recent American Thoracic Society statement. ${ }^{30}$

\section{Hyperinflation and relationship with COPD progression and co-morbidities}

Cross-sectional studies have demonstrated the presence of hyperinflation in milder stages of COPD, including dynamic hyperinflation during everyday activities. ${ }^{9,35-37}$ The early stages of hyperinflation and its progression may not be perceived by patients because of adaptive changes that compensate for the mechanical disadvantages (e.g. chest wall reconfiguration to accommodate overextended lungs and partially preserved function of the diaphragm despite operating at shortened muscle fibre length). ${ }^{20,38}$ Pathological changes in the muscle fibres of the diaphragm may be evident even in the milder stages of COPD. ${ }^{39}$

Hyperinflation, physical inactivity, and physical deconditioning may be related to the early development of comorbidities. Inactivity and muscle wasting are present in the earliest stages of $\mathrm{COPD}^{40-43}$ and may be important for the development of co-morbidities that can also occur in milder stages of COPD. ${ }^{44,45}$ For example, subclinical left ventricular dysfunction in milder COPD was found to be especially marked in patients with resting hyperinflation. ${ }^{46}$

The presence of hyperinflation may influence the progression of disease: resting hyperinflation (inspiratory capacity (IC)/TLC ratio $<25 \%$ ) has been associated with increased frequency of COPD exacerbations, ${ }^{47}$ and is an independent predictor of mortality in COPD. ${ }^{48}$ Of potential relevance, hyperinflation of the lung (assessed by quantitative computed tomography) was found to predict a rapid annual decline in forced expiratory volume in 1 second $\left(\mathrm{FEV}_{1}\right)$ in smokers with normal FEV $1 .{ }^{49}$ If, as recently shown, most of the decline in lung function occurs in milder disease, ${ }^{44,50-52}$ it may be hypothesised that some form of early intervention could prevent progressive functional deterioration and maintain organ function at a higher level.

\section{Measurement of hyperinflation in COPD}

Hyperinflation is difficult to measure with the investigations usually available in general practice. Relevant measures in COPD are functional residual capacity (the volume of air remaining in the lungs at the end of tidal expiration, an index of hyperinflation), and RV (the volume of air remaining in the lungs at the end of a maximal expiration, an index of air trapping), but both of these volume measurements require relatively sophisticated equipment (body plethysmography or spirometers with inert gas analysers). ${ }^{53}$

$\mathrm{IC}$ is often used as a surrogate measure of hyperinflation. This is the maximal volume of air that can be inspired after a quiet breath out, which is the difference between TLC and EELV. IC can be measured by simple spirometry using a closed-circuit system. ${ }^{53}$ Provided TLC remains constant, reduced resting IC indicates the presence of hyperinflation in the setting of expiratory flow limitation. $^{54}$ IC may not always be a reliable measure in conditions of severe hyperinflation or over time (when TLC changes). ${ }^{55,56}$ IC measurements during exercise reflect the 
increase in dynamic EELV and are more relevant physiologically with respect to exertional dyspnoea and exercise intolerance than resting $I C .{ }^{57} \mathrm{~A}$ recent study has shown that critical reduction of the inspiratory reserve volume (IRV; calculated as IC minus tidal volume), an index of 'the room to breathe', is more closely related to dyspnoea intensity during exercise in COPD than is the extent of air trapping per se. .8, $^{59}$

\section{Effect of treatment Treatment strategies}

A treatment that can bring about a reduction in EELV (and increase in IRV) should enable a patient to increase tidal volume more for a given effort, and thus lessen exertional dyspnoea. A treatment that improves expiratory flow limitation or decreases ventilatory demand will interrupt the vicious cycle of faster breathing and worsening hyperinflation. Thus, there is a range of treatment strategies ${ }^{60,61}$ that may be employed in different settings, from drug treatments and supplemental oxygen for patients with hypoxia or oxygen desaturation available in primary care, to assisted ventilation for hospitalised patients and surgical options for those with advanced emphysema.

Treatments that primarily decrease respiratory rate (reducing ventilatory demand) and increase ventilation include rehabilitative exercise training (pulmonary rehabilitation) and supplemental oxygen. Assisted ventilation counterbalances the negative effects of lung hyperinflation on the respiratory muscles. Treatment with bronchodilators primarily reduces flow limitation and improves lung emptying. Lung volume reduction surgery reduces EELV by favourably altering the elastic properties of the remaining lung, thus increasing lung emptying. The different mechanisms by which these interventions operate suggest that combinations would provide additional benefits, an example being the additive benefits shown by the combination of tiotropium (reduced hyperinflation) with supplemental oxygen (reduced ventilatory drive), ${ }^{62}$ and ipratropium bromide or supplemental oxygen with rehabilitation. ${ }^{63-65}$ This review will focus on the treatments that are available in the primary care setting, principally pharmacotherapy and exercise programmes.

\section{Pulmonary rehabilitation/exercise training}

Increasing activity levels is a key part of interrupting the downward spiral of disability and premature death for COPD patients. ${ }^{66}$ Pulmonary rehabilitation is a cornerstone in the comprehensive management of patients with COPD, and has recognised benefits for improved exercise endurance, dyspnoea, functional capacity, and quality of life (Table 1). ${ }^{67}$ The strength of evidence is reflected in its prominence in global and national COPD management guidelines. ${ }^{67-73}$ Pulmonary rehabilitation is currently the best way to improve quality of life in patients with COPD..$^{71,74}$ Comprehensive pulmonary rehabilitation programmes include exercise training, smoking cessation, nutrition counselling, and education. ${ }^{67}$

Exercise training improves skeletal muscle function and leads to less ventilatory requirement for a given work rate, which in
Table 1. Benefits of pulmonary rehabilitation in chronic obstructive pulmonary disease (COPD) ${ }^{67}$

\begin{tabular}{|c|c|}
\hline $\begin{array}{l}\text { Strength } \\
\text { of } \\
\text { evidence }\end{array}$ & Benefit \\
\hline \multirow[t]{5}{*}{ A } & Improves exercise capacity \\
\hline & Reduces the perceived intensity of breathlessness \\
\hline & Improves health status \\
\hline & Reduces number of hospitalisations and days in hospital \\
\hline & Reduces anxiety and depression associated with COPD \\
\hline \multirow[t]{5}{*}{ B } & $\begin{array}{l}\text { Upper limb strength/endurance training improves } \\
\text { arm function }\end{array}$ \\
\hline & Benefits persist beyond training period \\
\hline & Improves survival \\
\hline & Improves recovery after hospitalisation for exacerbation \\
\hline & Enhances the effect of long-acting bronchodilators \\
\hline C & $\begin{array}{l}\text { Respiratory muscle training can be beneficial } \\
\text { (dyspnoea, health status, exercise capacity), especially } \\
\text { when combined with general exercise training }\end{array}$ \\
\hline
\end{tabular}

$\mathrm{A}=$ randomised controlled trials (rich body of data), B=randomised controlled trials (limited body of data), C=non-randomised trials (observational studies).

turn may reduce dynamic hyperinflation, thus reducing exertional dyspnoea. ${ }^{70}$ Because exercise capacity is limited by both skeletal muscle fatigue and exertional dyspnoea due to dynamic hyperinflation, bronchodilator therapy may help improve the effectiveness of exercise training by lowering the barrier of activity-limiting dyspnoea and allowing patients to exercise their peripheral muscles to a greater degree. ${ }^{70,75}$ Practice guidelines recommend that optimal bronchodilator therapy should be given prior to exercise training. ${ }^{70}$ Even if a formal exercise programme is not available, patients should be encouraged to undertake regular exercise such as walking for 20 minutes a day. ${ }^{67}$

\section{Effect of pharmacotherapy on hyperinflation}

Evidence for the effects of pharmacotherapy on hyperinflation was gathered using the search strategy shown in Appendix 1 (online at www.thepcrj.org). Single-dose studies were not included.

\section{Bronchodilators}

Bronchodilators work by relaxing smooth muscle tone in the airways, leading to reduced respiratory muscle activity and improvements in ventilatory mechanics. ${ }^{76}$ In addition, reduced abdominal muscle activation and the consequent fall in gastric pressure following bronchodilator therapy may contribute to relieving the load on the respiratory system, similar to the relief provided by non-invasive ventilatory support. ${ }^{76-78}$ Bronchodilators have been associated with reduced airways resistance and elastic loading of the inspiratory muscles during constant work rate exercise. ${ }^{79}$ The lower operating lung volume allows patients to achieve the required alveolar ventilation during rest and exercise at a lower oxygen cost of breathing. By deflating the lungs, 


\begin{tabular}{|c|c|c|c|c|c|c|c|c|c|c|c|c|c|c|}
\hline $\begin{array}{l}\frac{y}{d} \\
\frac{5}{3} \\
\circ \\
0\end{array}$ & \multirow{4}{*}{ 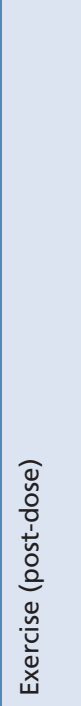 } & 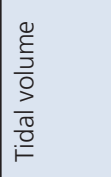 & 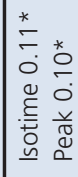 & 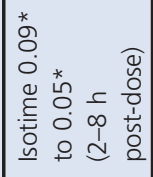 & 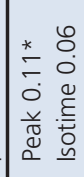 & 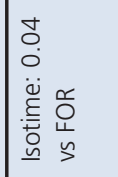 & I & 1 & 1 & 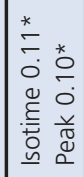 & 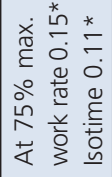 & 1 & 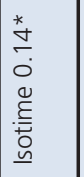 & \multirow{13}{*}{$\begin{array}{l} \\
\\
\end{array}$} \\
\hline 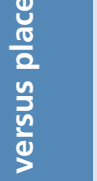 & & $\underline{\cup}$ & 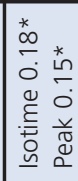 & 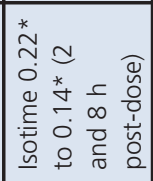 & 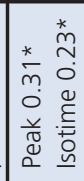 & 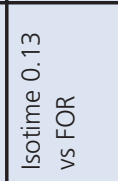 & 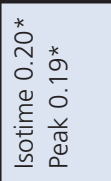 & 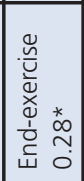 & 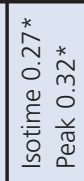 & 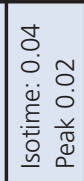 & 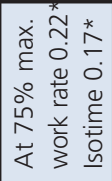 & 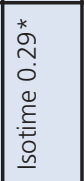 & 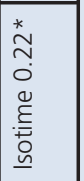 & \\
\hline 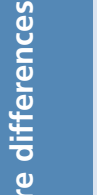 & & 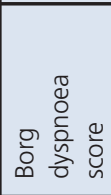 & 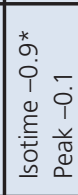 & 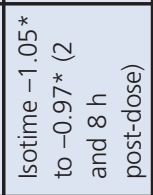 & 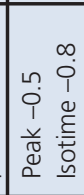 & 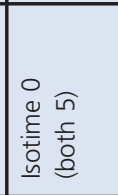 & 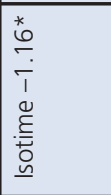 & 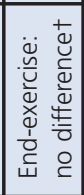 & 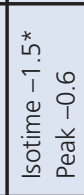 & 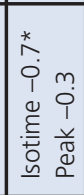 & 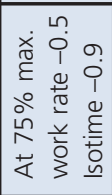 & 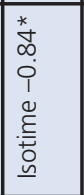 & 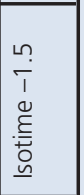 & \\
\hline 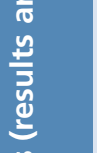 & & 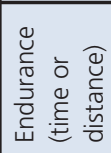 & \begin{tabular}{l}
$*$ \\
\multirow{n}{*}{} \\
$\stackrel{0}{0}$ \\
$\stackrel{0}{-}$
\end{tabular} & 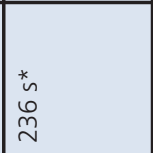 & $\begin{array}{l}\cdot \frac{c}{\varepsilon} \\
o \\
o \\
o\end{array}$ & 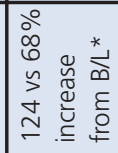 & 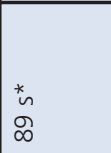 & 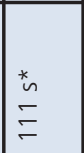 & 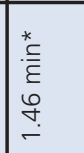 & \begin{tabular}{l}
$*$ \\
\multirow{0}{*}{} \\
$\underline{0}$
\end{tabular} & 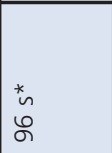 & $\tilde{v}$ & $\begin{array}{l}n \\
\stackrel{m}{n}\end{array}$ & \\
\hline 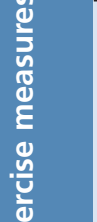 & 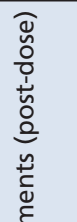 & $\begin{array}{l}\underline{\beth} \\
\underline{u}\end{array}$ & 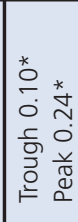 & 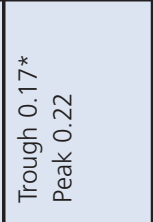 & $\stackrel{*}{\stackrel{*}{n}} \underset{\sim}{0}$ & 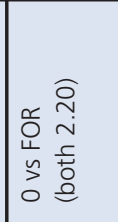 & \begin{tabular}{l}
$\stackrel{*}{N}$ \\
$\stackrel{1}{1}$ \\
$\stackrel{1}{N}$ \\
\multirow{N}{0}{} \\
0
\end{tabular} & 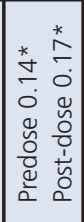 & 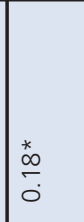 & $\begin{array}{l}+ \\
* \\
m \\
0 \\
0\end{array}$ & $\begin{array}{l}\stackrel{*}{n} \\
m \\
0 \\
0\end{array}$ & 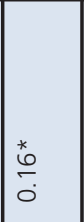 & $\begin{array}{l}* \\
\stackrel{*}{\infty} \\
\sim \\
0\end{array}$ & \\
\hline $\begin{array}{l}\frac{0}{0} \\
\frac{0}{0} \\
y \\
\frac{1}{E} \\
\frac{5}{0}\end{array}$ & 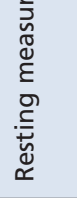 & 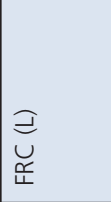 & 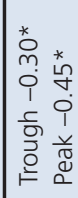 & 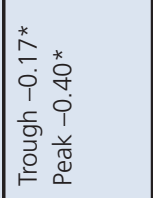 & $\begin{array}{l}\stackrel{*}{*} \\
\stackrel{2}{0} \\
i \\
\end{array}$ & 1 & 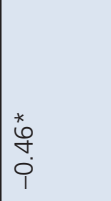 & 1 & $\begin{array}{l}\dot{\sigma} \\
m \\
i \\
i\end{array}$ & $\begin{array}{l}+ \\
* \\
\infty \\
\infty \\
0 \\
1\end{array}$ & 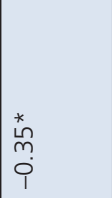 & 1 & & \\
\hline$\frac{\mathrm{g}}{\mathrm{s}}$ & 돈 & & $\begin{array}{l}\infty \\
0 \\
0 \\
0 \\
\propto 2\end{array}$ & $\begin{array}{l}\infty \\
0 \\
0 \\
0 \\
\propto 2\end{array}$ & $\begin{array}{l}\infty \\
0 \\
0 \\
\times \\
\check{x} \\
\check{x}\end{array}$ & $\begin{array}{l}0 \\
0 \\
0 \\
\times \\
\check{x} \\
\check{x}\end{array}$ & $\begin{array}{l}\stackrel{P}{x} \\
\propto x\end{array}$ & $\begin{array}{l}\varnothing \\
0 \\
\dot{x} \\
\dot{x} \\
\propto<\end{array}$ & $\begin{array}{l}\varnothing \\
0 \\
\dot{x} \\
\dot{x} \\
\propto َ\end{array}$ & $\begin{array}{l}\infty \\
0 \\
0 \\
0 \\
\propto 2\end{array}$ & $\begin{array}{l}\ddot{0} \\
\dot{0} \\
\dot{x} \\
\propto\end{array}$ & 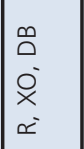 & $\begin{array}{l}\ddot{0} \\
0 \\
\dot{x} \\
\dot{x}\end{array}$ & \\
\hline $\begin{array}{l}\frac{5}{0} \\
\frac{0}{0} \\
\frac{1}{0} \\
0\end{array}$ & 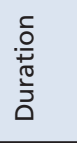 & & \begin{tabular}{|l}
$\tilde{y}$ \\
$\tilde{u}$ \\
$\sum_{3}$ \\
6
\end{tabular} & \begin{tabular}{|l}
$\tilde{y}$ \\
$\tilde{y}$ \\
$\sum_{0}$ \\
6
\end{tabular} & $\begin{array}{l}n \\
\frac{\pi}{0} \\
0 \\
\frac{1}{1} \\
n\end{array}$ & $\begin{array}{l}\tilde{v} \\
\stackrel{\tilde{u}}{\Delta} \\
\sum_{\sim} \\
\sim\end{array}$ & $\begin{array}{l}\tilde{u} \\
\tilde{y} \\
m \\
m\end{array}$ & $\begin{array}{l}\tilde{y} \\
\tilde{y} \\
\sum_{m}\end{array}$ & 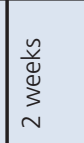 & \begin{tabular}{|l}
$\tilde{y}$ \\
$\tilde{u}$ \\
$\sum_{3}$ \\
6
\end{tabular} & $\begin{array}{l}\tilde{u} \\
\stackrel{\tilde{u}}{\tilde{u}} \\
\sim\end{array}$ & 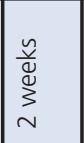 & $\begin{array}{l}\tilde{u} \\
\stackrel{\tilde{u}}{\tilde{N}} \\
\sim \\
\sim\end{array}$ & \\
\hline 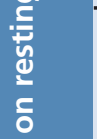 & 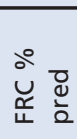 & & $\begin{array}{l}-\overline{0} \\
1 \\
0 \\
-0\end{array}$ & 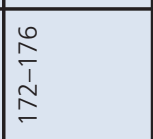 & $\stackrel{\Sigma}{\Sigma}$ & । & I & 1 & 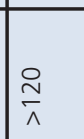 & 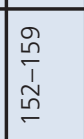 & $\underset{⿱}{\stackrel{N}{\Sigma}}$ & 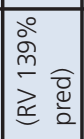 & 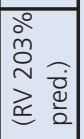 & \\
\hline $\begin{array}{l}\frac{n}{0} \\
\frac{0}{0} \\
\frac{0}{0}\end{array}$ & 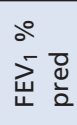 & & $\frac{\tilde{y}}{\frac{7}{\tau}}$ & $\stackrel{m}{q}$ & of & $\hat{\gamma}$ & ถิ & 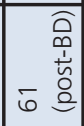 & 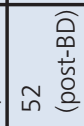 & బิ & $\approx$ & $\bar{m}$ & gr & \\
\hline $\begin{array}{l}\text { ్ } \\
\text { ț }\end{array}$ & c & & $\stackrel{\infty}{\infty}$ & $\underset{\sim}{\tilde{\sigma}}$ & $\stackrel{\infty}{\simeq}$ & $\stackrel{m}{m}$ & $\stackrel{\infty}{\circ}$ & 8 & $\lesssim$ & $\stackrel{\infty}{\infty}$ & $\stackrel{m}{\sim}$ & 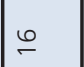 & $\bar{\sim}$ & \\
\hline 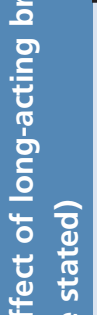 & 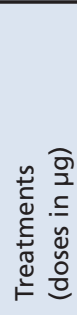 & & 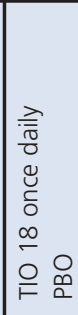 & 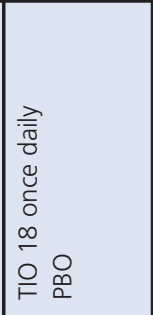 & 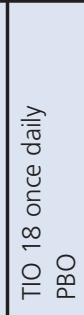 & 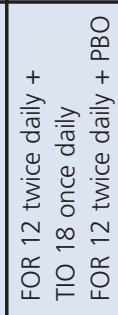 & 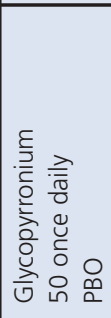 & 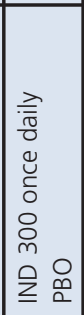 & 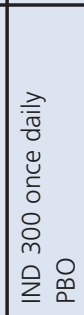 & 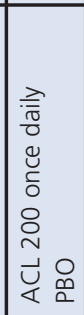 & 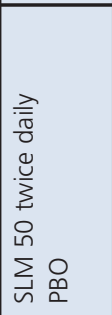 & 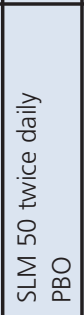 & 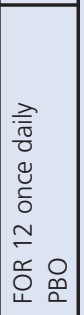 & \\
\hline 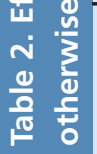 & 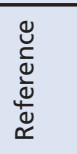 & & 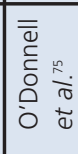 & 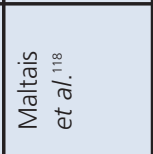 & 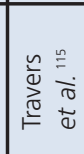 & 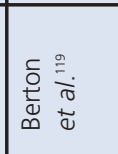 & 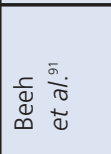 & 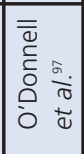 & 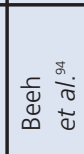 & 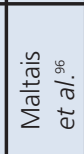 & 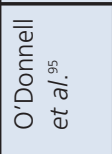 & 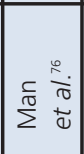 & 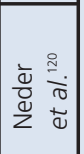 & \\
\hline
\end{tabular}


bronchodilators effectively improve ventilatory muscle performance resulting in greater tidal volume expansion. Thus, neuromechanical coupling is enhanced and dyspnoea is lessened. Reduction in absolute lung volume results in a delay in the time for end-inspiratory lung volume to reach the minimal dynamic IRV; the mechanical limitation of ventilation is postponed and exercise endurance time is prolonged. ${ }^{79}$ With short-acting inhaled bronchodilators, three multiple-dose studies reported improvements in lung volume and exercise measures with single or combined treatments ( $\beta_{2}$-agonist plus anticholinergic; $\beta 2$-agonist plus theophylline). ${ }^{80-82}$

Multiple-dose studies that have investigated the effects of long-acting bronchodilators on both resting hyperinflation and dynamic hyperinflation during exercise are summarised in Table 2 and Appendix 2 (available online at www.thepcrj.org). The results demonstrate improved IC throughout rest and exercise, in association with numerical or significant improvement in dyspnoea intensity ratings during exercise and increased exercise endurance (Table 2 and Appendix 2). Many other studies have investigated the effects on resting hyperinflation alone, and report improvements measured by IC and volume measurements during treatment with indacaterol, ${ }^{83}$ formoterol, ${ }^{84,85}$ salmeterol, ${ }^{86}$ tiotropium, ${ }^{55,87-90}$ and glycopyrronium. ${ }^{91}$ Furthermore, a study investigating the effects of bronchodilator therapy on ventilatory mechanics during exercise showed that significant reductions in dynamic hyperinflation and dyspnoea were paralleled by decreased respiratory muscle activity. ${ }^{76}$

There are few published comparisons of long-acting bronchodilators. Tiotropium was reported to be superior to salmeterol in improving resting volumes and exercise endurance, ${ }^{92,93}$ and indacaterol was reported to be superior to salmeterol for its effect on resting inspiratory capacity ${ }^{83}$

The additive effects of combining a longacting bronchodilator and pulmonary rehabilitation have been discussed above..$^{64,70}$ Because the beneficial effects of long-acting bronchodilators on exercise and associated hyperinflation are observed as early as day 1 of treatment, ${ }^{94-97}$ these agents may be particularly useful in the context of exercise programmes to provide patients with an initial 'boost' that may help them achieve successful rehabilitation.

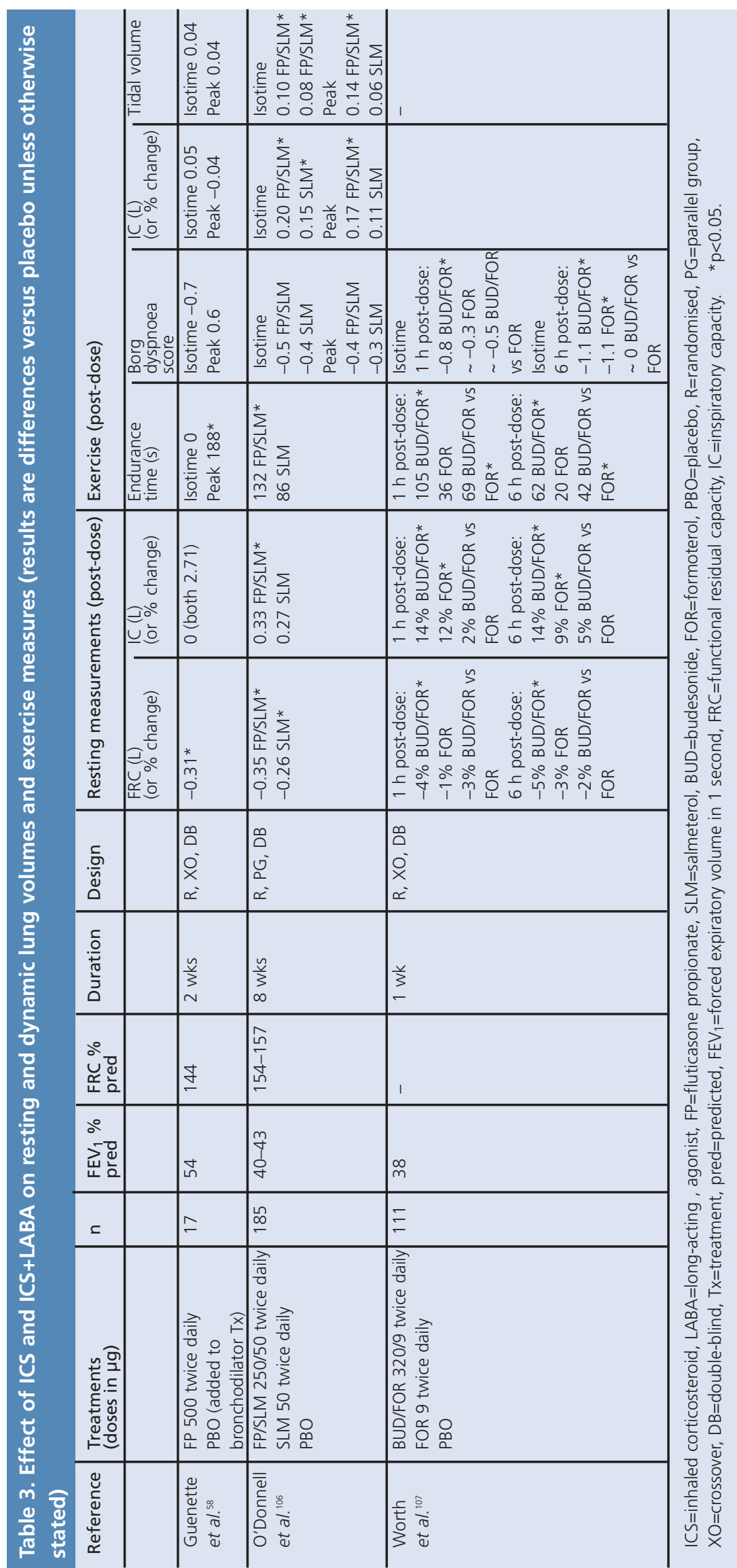


Combining two bronchodilators may extend the improvements seen with single agents - for example, resting IC was increased with indacaterol plus tiotropium compared with tiotropium alone ${ }^{98}$ or with formoterol plus tiotropium versus tiotropium alone. ${ }^{99-101}$ The addition of tiotropium to an inhaled corticosteroid (ICS)/long-acting $\beta_{2}$-agonist (LABA) combination has been reported to improve resting lung volumes compared with either individual component. ${ }^{102-104}$ The bronchodilator combination of tiotropium and salmeterol was more effective than an ICS/LABA for improving resting lung volumes in hyperinflated patients, although exercise endurance time was not significantly increased. ${ }^{105}$

\section{ICS and ICS/LABA combinations}

Two studies have compared the effects of ICS/LABA treatment with a LABA alone. O'Donnell et al. reported that fluticasone/salmeterol reduced resting and dynamic hyperinflation and increased exercise endurance time compared with placebo but not compared with salmeterol (Table 3 and Appendix 2). ${ }^{106}$ A later study with a higher ICS dose found that adding fluticasone to long-acting bronchodilator therapy decreased EELV during exercise without improvements in dyspnoea or IC. ${ }^{58}$ The budesonide/formoterol combination was reported to increase exercise endurance and reduce dynamic hyperinflation compared with both placebo and formoterol. ${ }^{107}$ The mechanism for an effect of ICS on hyperinflation is unclear, but may involve a direct local action on pulmonary cells or vasculature (e.g. vasoconstriction)..$^{58,107}$ The budesonide/ formoterol combination was reported to improve resting lung volumes more than salmeterol/fluticasone, ${ }^{108}$ and with a faster onset of effect. ${ }^{109,110}$

\section{Other treatments: roflumilast, theophylline, mucoactive treatment (acetylcysteine)}

The oral phosphodiesterase-4 inhibitor roflumilast had no effect on hyperinflation or exercise times compared with placebo after 12 weeks of treatment. ${ }^{111}$ Theophylline has been reported to reduce lung volumes and improve exercise endurance, ${ }^{112,113}$ possibly through improved respiratory muscle performance likely secondary to unmeasured lung volume reduction. ${ }^{14,116}$ Reduced hyperinflation and improved exercise endurance were reported following treatment with the mucoactive/antioxidant treatment acetylcysteine. ${ }^{117}$

\section{Conclusions}

While persistent and progressive expiratory flow limitation is the hallmark of COPD, its consequence - lung hyperinflation - importantly contributes to the exertional dyspnoea that limits activity and prevent patients from going about their normal everyday activities. Such inactivity can have a detrimental effect on physical conditioning and health-related quality of life. Acute increases in hyperinflation above the already increased resting level are likely to underlie the acute worsening of symptoms during increased activity and during exacerbations of COPD. Reducing hyperinflation and its consequences will have important benefits for COPD patients by interrupting the vicious cycle of dyspnoea, activity limitation, deconditioning, and impaired health-related quality of life. Some of the common co-morbidities in COPD start early in the disease and may be related to inactivity and physical deconditioning. ${ }^{44,45}$ It seems logical that the earlier the vicious cycle is interrupted, the better the outcome for patients, and there should be a lower threshold for initiating treatments appropriate to the stage of the disease (e.g. longacting bronchodilators and an exercise programme for patients with mild-to-moderate disease who experience persistent dyspnoea). Exercise and pulmonary rehabilitation have a valuable effect at all stages of the disease.

\section{Handling editor Anthony D'Urzo}

Acknowledgements Sarah Filcek (Circle Science, Tytherington, Cheshire, UK) assisted in preparing a first draft of the manuscript that was critically reviewed and revised by the authors, but did not meet the ICMJE criteria for authorship (www.icmje.org/).

Conflicts of interest Neither MT nor any member of his close family has any shares in pharmaceutical companies. In the last 3 years he has received speaker's honoraria for speaking at sponsored meetings from the following companies marketing respiratory and allergy products: AstraZeneca (AZ), Boehringer Ingleheim (BI), GlaxoSmithKline (GSK), MSD, Napp, Schering-Plough, Teva. He has received honoraria for attending advisory panels with Almirall, AZ, BI, Chiesi, GSK, MSD, Merck Respiratory, Schering-Plough, Teva, and Novartis. He has received sponsorship to attend international scientific meetings from GSK, MSD, AZ, and Mundipharma and he has received funding for research projects from GSK, MSD, and Napp. He held a research fellowship and is Chief Medical Advisor to Asthma UK. He is a member of the UK Department of Health Asthma Strategy Group and Home Oxygen Group. He is a member of the MHRA Respiratory and Allergy Expert Advisory Group, the BTS SIGN Asthma Guideline Group and the EPOS Rhinosinusitis Guideline Group. MT is an Associate Editor of the PCRJ, but was not involved in the editorial review of, nor the decision to publish, this article.

MD has received speaker fees from AZ, GSK, Boehringer-Pfizer, and Novartis; consulting fees from AZ, Boehringer-Pfizer, Dompé, GSK, Novartis, Nycomed, and Vectura; grant support from AZ, Boehringer-Pfizer, GSK, and Chiesi. He has no stock holdings in pharmaceutical companies and has never received grant support from the tobacco industry.

DEO has served on advisory boards for BI, Pfizer, GSK, Novartis and Nycomed; has received lecture fees from $\mathrm{BI}, \mathrm{AZ}$, Pfizer, and GSK; and has received industry-sponsored grants from: BI, GSK, Merck Frosst Canada, Novartis, and Pfizer.

Contributorship All authors were involved in the concept and design of this article. All authors revised the article critically for important intellectual content and gave their final approval of the version to be published.

Funding Preparation of the first draft of the manuscript was funded by Novartis Pharma AG (Basel, Switzerland). The authors did not receive any payment for producing this paper.

\section{References}

1. Buist AS, McBurnie MA, Vollmer WM, et al. International variation in the prevalence of COPD (the BOLD Study): a population-based prevalence study. Lancet 2007;370:741-50. http://dx.doi.org/10.1016/\$0140-6736(07)61377-4

2. East of England Public Health Observatory. Modelled estimate of prevalence of COPD in England. http://www.apho.org.uk/resource/item.aspx?RID=111122 (accessed 9 July 2012).

3. Hill K, Goldstein RS, Guyatt GH, et al. Prevalence and underdiagnosis of chronic obstructive pulmonary disease among patients at risk in primary care. CMA 2010;182:673-8. http://dx.doi.org/10.1503/cmaj.091784

4. Mannino DM, Buist AS. Global burden of COPD: risk factors, prevalence, and future trends. Lancet 2007;370:765-73. http://dx.doi.org/10.1016/S0140-6736(07)61380-4

5. Garcia-Aymerich J, Serra P, I, Mannino DM, et al. Lung function impairment, COPD hospitalisations and subsequent mortality. Thorax 2011;66:585-90. 
http://dx.doi.org/10.1136/thx.2010.152876

6. Jones PW. Activity limitation and quality of life in COPD. COPD 2007;4:273-8. http://dx.doi.org/10.1080/15412550701480265

7. O'Donnell DE, Webb KA. The major limitation to exercise performance in COPD is dynamic hyperinflation. J Appl Physiol 2008;105:753-5. http://dx.doi.org/10.1152/ japplphysiol.90336.2008b

8. Rennard S, Decramer M, Calverley PM, et al. Impact of COPD in North America and Europe in 2000: subjects' perspective of Confronting COPD International Survey. Eur Respir J 2002;20:799-805. http://dx.doi.org/10.1183/09031936.02.03242002

9. Ofir D, Laveneziana P, Webb KA, et al. Mechanisms of dyspnea during cycle exercise in symptomatic patients with GOLD stage I chronic obstructive pulmonary disease. Am J Respir Crit Care Med 2008;177:622-9. http://dx.doi.org/10.1164/rccm.200707-1064OC

10. Hogg JC, Chu F, Utokaparch $S$, et al. The nature of small-airway obstruction in chronic obstructive pulmonary disease. N Engl J Med 2004;350:2645-53. http://dx.doi.org/ 10.1056/NEJMoa032158

11. McDonough JE, Yuan R, Suzuki M, et al. Small-airway obstruction and emphysema in chronic obstructive pulmonary disease. N Engl J Med 2011;365:1567-75. http://dx.doi.org/10.1056/NEJMoa1106955

12. O'Donnell DE, Parker CM. COPD exacerbations . 3: Pathophysiology. Thorax 2006;61:354-61. http://dx.doi.org/10.1136/thx.2005.041830

13. Decramer M. Respiratory muscles in COPD: regulation of trophical status. Verh $K$ Acad Geneeskd Belg 2001;63:577-602.

14. Decramer M. Pulmonary rehabilitation 2007: from bench to practice and back. Clin Invest Med 2008;31:E312-E318.

15. Gosselink R, Troosters T, Decramer M. Peripheral muscle weakness contributes to exercise limitation in COPD. Am J Respir Crit Care Med 1996;153:976-80.

16. O'Donnell DE. Hyperinflation, dyspnea, and exercise intolerance in chronic obstructive pulmonary disease. Proc Am Thorac Soc 2006;3:180-4. http://dx.doi.org/ 10.1513/pats.200508-093DO

17. McKenzie DK, Butler JE, Gandevia SC. Respiratory muscle function and activation in chronic obstructive pulmonary disease. J Appl Physiol 2009;107:621-9. http://dx.doi.org/10.1152/japplphysiol.00163.2009

18. Cassart M, Pettiaux N, Gevenois PA, et al. Effect of chronic hyperinflation on diaphragm length and surface area. Am J Respir Crit Care Med 1997;156:504-08.

19. Gorman RB, McKenzie DK, Pride NB, et al. Diaphragm length during tidal breathing in patients with chronic obstructive pulmonary disease. Am J Respir Crit Care Med 2002;166:1461-9. http://dx.doi.org/10.1164//rccm.200111-0870C

20. Similowski $T$, Yan $S$, Gauthier $A P$, et al. Contractile properties of the human diaphragm during chronic hyperinflation. N Engl J Med 1991;325:917-23. http://dx.doi.org/10.1056/NEJM199109263251304

21. Klimathianaki M, Vaporidi K, Georgopoulos D. Respiratory muscle dysfunction in COPD: from muscles to cell. Curr Drug Targets 2011;12:478-88. http://dx.doi.org/10.2174/138945011794751474

22. Parker CM, Voduc N, Aaron SD, et al. Physiological changes during symptom recovery from moderate exacerbations of COPD. Eur Respir J 2005;26:420-8. http://dx.doi.org/10.1183/09031936.05.00136304

23. Stevenson NJ, Walker PP, Costello RW, Calverley PM. Lung mechanics and dyspnea during exacerbations of chronic obstructive pulmonary disease. Am J Respir Crit Care Med 2005;172:1510-6. http://dx.doi.org/10.1164/rccm.200504-5950C

24. Celli BR, Barnes PJ. Exacerbations of chronic obstructive pulmonary disease. Eur Respir J 2007;29:1224-38. http://dx.doi.org/10.1183/09031936.00109906

25. Vogelmeier C, Hederer B, Glaab $T$, et al. Tiotropium versus salmeterol for the prevention of exacerbations of COPD. N Engl J Med 2011:364:1093-103. http://dx.doi.org/10.1056/NEJMoa1008378

26. Washko GR, Fan VS, Ramsey SD, et al. The effect of lung volume reduction surgery on chronic obstructive pulmonary disease exacerbations. Am J Respir Crit Care Med 2008;177:164-9. http://dx.doi.org/10.1164/rccm.200708-11940C

27. Wedzicha JA, Calverley PM, Seemungal TA, et al. The prevention of chronic obstructive pulmonary disease exacerbations by salmeterol/fluticasone propionate or tiotropium bromide. Am J Respir Crit Care Med 2008;177:19-26. http://dx.doi.org/10.1164/rccm.200707-9730C

28. Coussa ML, Guerin C, Eissa NT, et al. Partitioning of work of breathing in mechanically ventilated COPD patients. J Appl Physiol 1993;75:1711-19.

29. O'Donnell DE, Ora J, Webb KA, et al. Mechanisms of activity-related dyspnea in pulmonary diseases. Respir Physiol Neurobiol 2009;167:116-32. http://dx.doi.org/ 10.1016/j.resp.2009.01.010

30. Parshall MB, Schwartzstein RM, Adams L, et al. An official American Thoracic Society statement: update on the mechanisms, assessment, and management of dyspnea. Am J Respir Crit Care Med 2012;185:435-52. http://dx.doi.org/10.1164/ rccm.201111-2042ST

31. Laveneziana P, Webb KA, Ora J, et al. Evolution of dyspnea during exercise in chronic obstructive pulmonary disease: impact of critical volume constraints. Am J Respir Crit Care Med 2011;184:1367-73. http://dx.doi.org/10.1164/rccm.201106-11280C

32. O'Donnell DE, Guenette JA, Maltais F, Webb KA. Decline of resting inspiratory capacity in COPD: the impact on breathing pattern, dyspnea, and ventilatory capacity during exercise. Chest 2012;141:753-62. http://dx.doi.org/10.1378/chest.11-0787

33. Williams M, Cafarella P, Olds T, et al. The language of breathlessness differentiates between patients with COPD and age-matched adults. Chest 2008;134:489-96. http://dx.doi.org/10.1378/chest.07-2916

34. Williams $\mathrm{M}$, Cafarella $\mathrm{P}$, Olds $\mathrm{T}$, et al. Affective descriptors of the sensation of breathlessness are more highly associated with severity of impairment than physical descriptors in people with COPD. Chest 2010;138:315-22. http://dx.doi.org/ 10.1378/chest.09-2498

35. Deesomchok A, Webb KA, Forkert L, et al. Lung hyperinflation and its reversibility in patients with airway obstruction of varying severity. COPD 2010;7:428-37. http://dx.doi.org/10.3109/15412555.2010.528087

36. Garcia-Rio F, Lores V, Mediano O, et al. Daily physical activity in patients with chronic obstructive pulmonary disease is mainly associated with dynamic hyperinflation. Am J Respir Crit Care Med 2009;180:506-12. http://dx.doi.org/10.1164/rccm.200812$18730 \mathrm{C}$

37. Hannink JD, van Helvoort HA, Dekhuijzen PN, Heijdra YF. Dynamic hyperinflation during daily activities: does COPD global initiative for chronic obstructive lung disease stage matter? Chest 2010;137:1116-21. http://dx.doi.org/10.1378/chest.09-1847

38. Levine S, Kaiser L, Leferovich J, Tikunov B. Cellular adaptations in the diaphragm in chronic obstructive pulmonary disease. N Engl J Med 1997;337:1799-806. http://dx.doi.org/10.1056/NEJM199712183372503

39. Ottenheijm CA, Heunks LM, Dekhuijzen RP. Diaphragm adaptations in patients with COPD. Respir Res 2008;9:12. http://dx.doi.org/10.1186/1465-9921-9-12

40. Pitta F, Troosters T, Spruit MA, et al. Characteristics of physical activities in daily life in chronic obstructive pulmonary disease. Am J Respir Crit Care Med 2005;171:972-7. http://dx.doi.org/10.1164/rccm.200407-8550C

41. Shrikrishna D, Patel M, Tanner RJ, et al. Quadriceps wasting and physical inactivity in patients with COPD. Eur Respir J 2012;40:1115-22. http://dx.doi.org/10.1183/ 09031936.00170111

42. Watz H, Waschki B, Boehme C, et al. Extrapulmonary effects of chronic obstructive pulmonary disease on physical activity: a cross-sectional study. Am J Respir Crit Care Med 2008;177:743-51. http://dx.doi.org/10.1164/rccm.200707-10110C

43. Watz $\mathrm{H}$, Waschki B, Meyer T, Magnussen $\mathrm{H}$. Physical activity in patients with COPD. Eur Respir J 2009;33:262-72. http://dx.doi.org/10.1183/09031936.00024608

44. Decramer M, Cooper CB. Treatment of COPD: the sooner the better? Thorax 2010;65:837-41. http://dx.doi.org/10.1136/thx.2009.133355

45. Decramer M, Rennard S, Troosters $\mathrm{T}$, et al. COPD as a lung disease with systemic consequences: clinical impact, mechanisms, and potential for early intervention. COPD 2008;5:235-56. http://dx.doi.org/10.1080/15412550802237531

46. Malerba $M$, Ragnoli $B$, Salameh $M$, et al. Sub-clinical left ventricular diastolic dysfunction in early stage of chronic obstructive pulmonary disease. J Biol Regul Homeost Agents 2011;25:443-51.

47. Zaman M, Mahmood S, Altayeh A. Low inspiratory capacity to total lung capacity ratio is a risk factor for chronic obstructive pulmonary disease exacerbation. Am J Med Sci 2010;339:411-14.

48. Casanova C, Cote C, de Torres JP, et al. Inspiratory-to-total lung capacity ratio predicts mortality in patients with chronic obstructive pulmonary disease. Am J Respir Crit Care Med 2005;171:591-7. http://dx.doi.org/10.1164/rccm.200407-8670C

49. Yuan $\mathrm{R}$, Hogg JC, Pare PD, et al. Prediction of the rate of decline in FEV(1) in smokers using quantitative computed tomography. Thorax 2009;64:944-9. http://dx.doi.org/10.1136/thx.2008.112433

50. Casanova C, de Torres JP, guirre-Jaime A, et al. The progression of chronic obstructive pulmonary disease is heterogeneous: the experience of the BODE cohort. Am J Respir Crit Care Med 2011;184:1015-21. http://dx.doi.org/10.1164/rccm.201105$08310 \mathrm{C}$

51. Mohamed Hoesein FA, Zanen P, Boezen HM, et al. Lung function decline in heavy 
male smokers relates to baseline airflow obstruction severity. Chest 2012 Jun 21 [Epub ahead of print]. http://dx.doi.org/10.1378/chest.11-2837

52. Vestbo J, Edwards LD, Scanlon PD, et al. Changes in forced expiratory volume in 1 second over time in COPD. N Engl J Med 2011;365:1184-92. http://dx.doi.org/10.1056/NEJMoa1105482

53. Cazzola M, MacNee W, Martinez FJ, et al. Outcomes for COPD pharmacological trials: from lung function to biomarkers. Eur Respir J 2008;31:416-69. http://dx.doi.org/10.1183/09031936.00099306

54. Diaz O, Villafranca C, Ghezzo $\mathrm{H}$, et al. Role of inspiratory capacity on exercise tolerance in COPD patients with and without tidal expiratory flow limitation at rest. Eur Respir J 2000;16:269-75. http://dx.doi.org/10.1034/j.1399-3003.2000.16b14.x

55. Celli $B$, ZuWallack R, Wang $S$, Kesten $S$. Improvement in resting inspiratory capacity and hyperinflation with tiotropium in COPD patients with increased static lung volumes. Chest 2003;124:1743-8. http://dx.doi.org/10.1378/chest.124.5.1743

56. O'Donnell DE, Laveneziana P. Physiology and consequences of lung hyperinflation in COPD. Eur Respir Rev 2006;15:61-7. http://dx.doi.org/10.1183/ 09059180.00010002

57. O'Donnell DE, Lam M, Webb KA. Spirometric correlates of improvement in exercise performance after anticholinergic therapy in chronic obstructive pulmonary disease. Am J Respir Crit Care Med 1999;160:542-9

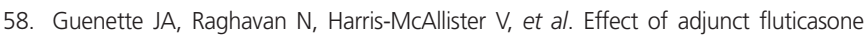
propionate on airway physiology during rest and exercise in COPD. Respir Med 2011;105:1836-45. http://dx.doi.org/10.1016/j.rmed.2011.08.021

59. Luo YM, Hopkinson NS, Polkey MI. Tough at the top: must end-expiratory lung volume make way for end-inspiratory lung volume? Eur Respir J 2012;40:283-5. http://dx.doi.org/10.1183/09031936.00021912

60. Casaburi R, Porszasz J. Reduction of hyperinflation by pharmacologic and other interventions. Proc Am Thorac Soc 2006;3:185-9. http://dx.doi.org/10.1513/ pats.200508-095DO

61. Puente-Maestu L, Stringer WW. Hyperinflation and its management in COPD. Int J Chron Obstruct Pulmon Dis 2006;1:381-400. http://dx.doi.org/10.2147/ copd.2006.1.4.381

62. Peters MM, Webb KA, O'Donnell DE. Combined physiological effects of bronchodilators and hyperoxia on exertional dyspnoea in normoxic COPD. Thorax 2006;61:559-67. http://dx.doi.org/10.1136/thx.2005.053470

63. Ambrosino N, Foglio K, Balzano G, et al. Tiotropium and exercise training in COPD patients: effects on dyspnea and exercise tolerance. Int J Chron Obstruct Pulmon Dis 2008;3:771-80

64. Casaburi R, Kukafka D, Cooper CB, et al. Improvement in exercise tolerance with the combination of tiotropium and pulmonary rehabilitation in patients with COPD. Chest 2005;127:809-17. http://dx.doi.org/10.1378/chest.127.3.809

65. Voduc N, Tessier C, Sabri E, et al. Effects of oxygen on exercise duration in chronic obstructive pulmonary disease patients before and after pulmonary rehabilitation. Can Respir J 2010;17:e14-19.

66. Casaburi R. Activity promotion: a paradigm shift for chronic obstructive pulmonary disease therapeutics. Proc Am Thorac Soc 2011;8:334-7. http://dx.doi.org/10.1513/ pats.201101-001RM

67. Global Initiative for Chronic Obstructive Lung Disease (GOLD). Global strategy for the diagnosis, management, and prevention of chronic obstructive pulmonary disease. www goldcopd com (accessed 14 June 2012).

68. Bellamy D, Bouchard J, Henrichsen S, et al. International Primary Care Respiratory Group (IPCRG) guidelines: management of chronic obstructive pulmonary disease (COPD). Prim Care Respir J 2006;15:48-57. http://dx.doi.org/10.1016/ j.pcri.2005.11.003

69. Marciniuk DD, Brooks D, Butcher $S$, et al. Optimizing pulmonary rehabilitation in chronic obstructive pulmonary disease--practical issues: a Canadian Thoracic Society clinical practice guideline. Can Respir J 2010;17:159-68.

70. Nici L, Donner C, Wouters E, et al. American Thoracic Society/European Respiratory Society statement on pulmonary rehabilitation. Am J Respir Crit Care Med 2006;173:1390-413. http://dx.doi.org/10.1164/rccm.200508-1211ST

71. Puhan MA, Gimeno-Santos E, Scharplatz M, et al. Pulmonary rehabilitation following exacerbations of chronic obstructive pulmonary disease. Cochrane Database Syst Rev 2011;CD005305

72. Qaseem A, Wilt TJ, Weinberger SE, et al. Diagnosis and management of stable chronic obstructive pulmonary disease: a clinical practice guideline update from the American College of Physicians, American College of Chest Physicians, American
Thoracic Society, and European Respiratory Society. Ann Intern Med 2011;155:17991.

73. Ries AL, Bauldoff GS, Carlin BW, et al. Pulmonary rehabilitation: Joint ACCP/AACVPR evidence-based clinical practice guidelines. Chest 2007;131:4S-42S http://dx.doi.org/10.1378/chest.06-2418

74. Lacasse Y, Goldstein R, Lasserson TJ, Martin S. Pulmonary rehabilitation for chronic obstructive pulmonary disease. Cochrane Database Syst Rev 2006;CD003793.

75. O'Donnell DE, Fluge T, Gerken F, et al. Effects of tiotropium on lung hyperinflation, dyspnoea and exercise tolerance in COPD. Eur Respir J 2004;23:832-40. http://dx.doi.org/10.1183/09031936.04.00116004

76. Man WD, Mustfa N, Nikoletou D, et al. Effect of salmeterol on respiratory muscle activity during exercise in poorly reversible COPD. Thorax 2004;59:471-6. http://dx.doi.org/10.1136/thx.2003.019620

77. Duranti R, Misuri G, Gorini M, et al. Mechanical loading and control of breathing in patients with severe chronic obstructive pulmonary disease. Thorax 1995;50:127-33. http://dx.doi.org/10.1136/thx.50.2.127

78. Kyroussis D, Polkey Ml, Hamnegard $\mathrm{CH}$, et al. Respiratory muscle activity in patients with COPD walking to exhaustion with and without pressure support. Eur Respir J 2000;15:649-55. http://dx.doi.org/10.1034/j.1399-3003.2000.15d05.x

79. O'Donnell DE, Banzett RB, Carrieri-Kohlman V, et al. Pathophysiology of dyspnea in chronic obstructive pulmonary disease: a roundtable. Proc Am Thorac Soc 2007;4:145-68. http://dx.doi.org/10.1513/pats.200611-159CC

80. Eiser $\mathrm{N}$, Angus $\mathrm{K}, \mathrm{McH}$ ale $\mathrm{S}$. The role of domiciliary nebulizers in managing patients with severe COPD. Respir Med 2001;95:265-74. http://dx.doi.org/10.1053/ rmed.2001.1032

81. Shioya T, Satake M, Sato $K$, et al. Long-term effect of the beta2-receptor agonist procaterol on daily life performance and exercise capacity in patients with stable chronic obstructive pulmonary disease. Clinical study with special reference to health-related quality of life and activities of daily living. Arzneimittelforschung 2008:58:24-8.

82. Thomas P, Pugsley JA, Stewart JH. Theophylline and salbutamol improve pulmonary function in patients with irreversible chronic obstructive pulmonary disease. Chest 1992;101:160-5. http://dx.doi.org/10.1378/chest.101.1.160

83. Laforce C, Aumann J, de Teresa PL, et al. Sustained 24-hour efficacy of once daily indacaterol (300 mug) in patients with chronic obstructive pulmonary disease: a randomized, crossover study. Pulm Pharmacol Ther 2011;24:162-8. http://dx.doi.org/10.1016/j.pupt.2010.06.005

84. Brusasco V, Canonica GW, Dal NR, et al. Formoterol by pressurized metered-dose aerosol or dry powder on airway obstruction and lung hyperinflation in partially reversible COPD. J Aerosol Med Pulm Drug Deliv 2011;24:235-43. http://dx.doi.org/10.1089/jamp.2010.0862

85. Hanania NA, Donohue JF, Nelson $\mathrm{H}$, et al. The safety and efficacy of arformoterol and formoterol in COPD. COPD 2010;7:17-31. http://dx.doi.org/10.3109/ 15412550903499498

86. Stockley RA, Chopra N, Rice L. Addition of salmeterol to existing treatment in patients with COPD: a 12 month study. Thorax 2006;61:122-8. http://dx.doi.org/10.1136/thx.2004.033266

87. Brashier $B$, Dhembare $P$, Jantikar $A$, et al. Tiotropium administered by a pressurized metered dose inhaler (pMDI) and spacer produces a similar bronchodilator response as that administered by a Rotahaler in adult subjects with stable moderate-to-severe COPD. Respir Med 2007;101:2464-71. http://dx.doi.org/10.1016/ j.rmed.2007.07.006

88. Stepanian IE, Khmel'kova NG, Belin-Atarac B. [The efficacy of tiotropium bromide (Spiriva) in the treatment of patients with chronic obstructive pulmonary disease of varying severity: results of the Russian trial]. Ter Arkh 2010;82:46-51.

89. Tanabe N, Muro S, Oguma $T$, et al. Computed tomography assessment of pharmacological lung volume reduction induced by bronchodilators in COPD. COPD 2012;9:401-08

90. Verkindre C, Bart F, Aguilaniu B, et al. The effect of tiotropium on hyperinflation and exercise capacity in chronic obstructive pulmonary disease. Respiration 2006;73:4207. http://dx.doi.org/10.1159/000089655

91. Beeh KM, Singh D, Di SL, Drollmann A. Once-daily NVA237 improves exercise tolerance from the first dose in patients with COPD: the GLOW3 trial. Int J Chron Obstruct Pulmon Dis 2012;7:503-13. http://dx.doi.org/10.2147/COPD.S32451

92. Fujimoto $K$, Kitaguchi $Y$, Kanda $S$, et al. Comparison of efficacy of long-acting bronchodilators in emphysema dominant and emphysema nondominant chronic 
obstructive pulmonary disease. Int J Chron Obstruct Pulmon Dis 2011;6:219-27. http://dx.doi.org/10.2147/COPD.S18461

93. van der Vaart $H$, Postma DS, Grevink $R$, et al. Bronchodilation improves endurance but not muscular efficiency in chronic obstructive pulmonary disease. Int J Chron Obstruct Pulmon Dis 2011;6:229-35. http://dx.doi.org/10.2147/COPD.S17482

94. Beeh KM, Wagner F, Khindri S, Drollmann AF. Effect of indacaterol on dynamic lung hyperinflation and breathlessness in hyperinflated patients with COPD. COPD 2011;8:340-5. http://dx.doi.org/10.3109/15412555.2011.594464

95. O'Donnell DE, Voduc N, Fitzpatrick M, Webb KA. Effect of salmeterol on the ventilatory response to exercise in chronic obstructive pulmonary disease. Eur Respir J 2004;24:86-94. http://dx.doi.org/10.1183/09031936.04.00072703

96. Maltais F, Celli B, Casaburi R, et al. Aclidinium bromide improves exercise endurance and lung hyperinflation in patients with moderate to severe COPD. Respir Med 2011;105:580-7. http://dx.doi.org/10.1016/j.rmed.2010.11.019

97. O'Donnell DE, Casaburi R, Vincken W, et al. Effect of indacaterol on exercise endurance and lung hyperinflation in COPD. Respir Med 2011;105:1030-6. http://dx.doi.org/10.1016/j.rmed.2011.03.014

98. Mahler DA, D'Urzo A, Bateman ED, et al. Concurrent use of indacaterol plus tiotropium in patients with COPD provides superior bronchodilation compared with tiotropium alone: a randomised, double-blind comparison. Thorax 2012;67:781-8. http://dx.doi.org/10.1136/thoraxjnl-2011-201140

99. Hanania NA, Boota A, Kerwin E, et al. Efficacy and safety of nebulized formoterol as add-on therapy in COPD patients receiving maintenance tiotropium bromide: results from a 6-week, randomized, placebo-controlled, clinical trial. Drugs 2009;69:120516. http://dx.doi.org/10.2165/00003495-200969090-00005

100. Tashkin DP, Donohue JF, Mahler DA, et al. Effects of arformoterol twice daily, tiotropium once daily, and their combination in patients with COPD. Respir Med 2009;103:516-24. http://dx.doi.org/10.1016/j.rmed.2008.12.014

101. van Noord JA, Aumann JL, Janssens E, et al. Effects of tiotropium with and without formoterol on airflow obstruction and resting hyperinflation in patients with COPD. Chest 2006;129:509-17. http://dx.doi.org/10.1378/chest.129.3.509

102. Perng DW, Wu CC, Su KC, et al. Additive benefits of tiotropium in COPD patients treated with long-acting beta agonists and corticosteroids. Respirology 2006;11:598602. http://dx.doi.org/10.1111/j.1440-1843.2006.00889.x

103. Singh D, Brooks J, Hagan G, et al. Superiority of "triple" therapy with salmeterol/fluticasone propionate and tiotropium bromide versus individual components in moderate to severe COPD. Thorax 2008;63:592-8. http://dx.doi.org/ 10.1136/thx.2007.087213

104. Welte T, Miravitlles M, Hernandez P, et al. Efficacy and tolerability of budesonide/formoterol added to tiotropium in patients with chronic obstructive pulmonary disease. Am J Respir Crit Care Med 2009;180:741-50. http://dx.doi.org/10.1164/rccm.200904-04920C

105. Magnussen $H$, Paggiaro $P$, Schmidt $H$, et al. Effect of combination treatment on lung volumes and exercise endurance time in COPD. Respir Med 2012;106:1413-20. http://dx.doi.org/10.1016/j.rmed.2012.05.011

106. O'Donnell DE, Sciurba F, Celli B, et al. Effect of fluticasone propionate/salmeterol on lung hyperinflation and exercise endurance in COPD. Chest 2006;130:647-56. http://dx.doi.org/10.1378/chest.130.3.647

107. Worth $\mathrm{H}$, Forster K, Eriksson G, et al. Budesonide added to formoterol contributes to improved exercise tolerance in patients with COPD. Respir Med 2010;104:1450-9. http://dx.doi.org/10.1016/j.rmed.2010.07.006

108. Tzani P, Crisafulli E, Nicolini G, et al. Effects of beclomethasone/formoterol fixed combination on lung hyperinflation and dyspnea in COPD patients. Int J Chron Obstruct Pulmon Dis 2011;6:503-09.

109. Celli BR, Tashkin DP, Rennard SI, et al. Bronchodilator responsiveness and onset of effect with budesonide/formoterol pMDI in COPD. Respir Med 2011;105:1176-88. http://dx.doi.org/10.1016/j.rmed.2011.02.020

110. Lindberg A, Szalai Z, Pullerits T, Radeczky E. Fast onset of effect of budesonide/formoterol versus salmeterol/fluticasone and salbutamol in patients with chronic obstructive pulmonary disease and reversible airway obstruction. Respirology 2007;12:732-9. http://dx.doi.org/10.1111/j.1440-1843.2007.01132.x

111. O'Donnell DE, Bredenbroker D, Brose M, Webb KA. Physiological effects of roflumilast at rest and during exercise in COPD. Eur Respir J 2012;39:1104-12. http://dx.doi.org/10.1183/09031936.00096511

112. Dreyse J, Silva F, Diaz O, et al. [Clinical and functional benefits of adding theophylline to a standard treatment with short acting bronchodilators in patients with COPD]. Rev Med Chil 2005;133:1211-19. http://dx.doi.org/10.4067/S003498872005001000010

113. Waterhouse JC, Pritchard SM, Howard P. Hyperinflation, trapped gas and theophylline in chronic obstructive pulmonary disease. Monaldi Arch Chest Dis 1993;48:126-9.

114. Murciano D, Aubier M, Lecocguic $Y$, Pariente R. Effects of theophylline on diaphragmatic strength and fatigue in patients with chronic obstructive pulmonary disease. $N$ Engl J Med 1984;311:349-53. http://dx.doi.org/10.1056/ NEJM198408093110601

115. Travers J, Laveneziana P, Webb KA, et al. Effect of tiotropium bromide on the cardiovascular response to exercise in COPD. Respir Med 2007;101:2017-24.

116. Murciano D, Auclair MH, Pariente R, Aubier M. A randomized, controlled trial of theophylline in patients with severe chronic obstructive pulmonary disease. N Engl J Med 1989;320:1521-5. http://dx.doi.org/10.1056/NEJM198906083202304

117. Stav D, Raz M. Effect of N-acetylcysteine on air trapping in COPD: a randomized placebo-controlled study. Chest 2009;136:381-6. http://dx.doi.org/10.1378/ chest.09-0421

118. Maltais F, Hamilton A, Marciniuk D, et al. Improvements in symptom-limited exercise performance over $8 \mathrm{~h}$ with once-daily tiotropium in patients with COPD. Chest 2005;128:1168-78. http://dx.doi.org/10.1378/chest.128.3.1168

119. Berton DC, Reis $M$, Siqueira AC, et al. Effects of tiotropium and formoterol on dynamic hyperinflation and exercise endurance in COPD. Respir Med 2010;104:1288-96. http://dx.doi.org/10.1016/j.rmed.2010.05.017

120. Neder JA, Fuld JP, Overend T, et al. Effects of formoterol on exercise tolerance in severely disabled patients with COPD. Respir Med 2007;101:2056-64. http://dx.doi.org/10.1016/j.rmed.2007.06.006

Available online at http://www.thepcrj.org 


\section{Appendix 1.}

Search no. 5 was taken as starting point, followed by selection based on relevance of abstracts; narrower searches were thought possibly restrictive. Results were cross-checked by conducting separate searches for 'inspiratory capacity' and individual drug names. Search last updated 11 September 2012 (no starting date limit).

\begin{tabular}{|c|c|c|}
\hline Search & Query & Items found \\
\hline \#10 & Search \#1 AND \#4 AND \#9 therapeutics subset & 521 \\
\hline \#9 & $\begin{array}{l}\text { Search "therapeutics" [MeSH] OR "therapy" [tiab] OR "treatment" [tiab] OR } \\
\text { "treatments" [tiab] }\end{array}$ & 5272386 \\
\hline \#6 & Search \#5 NOT \#3 & 768 \\
\hline \#5 & Search \#1 AND \#4 all results & 1069 \\
\hline \#4 & $\begin{array}{l}\text { Search "Functional Residual Capacity" [MeSH] OR "Expiratory Reserve } \\
\text { Volume"[MeSH] OR "Residual Volume"[MeSH] OR "air trapping" OR "air- } \\
\text { trapping" OR "gas trapping" OR "gas-trapping" OR "hyperaerated" OR } \\
\text { "hyperaeration" OR "hyperinflated" OR "hyperinflation" OR "functional } \\
\text { residual capacity" OR "residual volume" OR "over distended" OR "over- } \\
\text { distended" OR "over distension" OR "over-distension" OR "overinflated" } \\
\text { OR "over-inflated" OR "overinflation" OR "over-inflation" }\end{array}$ & 10017 \\
\hline \#3 & Search \#1 AND \#2 effects subset & 301 \\
\hline$\# 2$ & $\begin{array}{l}\text { Search "Functional Residual Capacity/drug effects" [MeSH] OR "Expiratory } \\
\text { Reserve Volume/drug effects"[MeSH] OR "Residual Volume/drug } \\
\text { effects" [MeSH] OR (("air trapping" OR "air-trapping" OR "gas trapping" } \\
\text { OR "gas-trapping" OR "hyperaerated" OR "hyperaeration" OR } \\
\text { "hyperinflated" OR "hyperinflation" OR "functional residual capacity" OR } \\
\text { "residual volume" OR "over distended" OR "over-distended" OR "Over } \\
\text { distension" OR "over-distension" OR "overinflated" OR "over-inflated" OR } \\
\text { "overinflation" OR "over-inflation") AND ("effects of treatment" OR } \\
\text { "effects of therapy" OR "therapeutic effect" OR "treatment effect" OR } \\
\text { "therapeutic effects" OR "treatment effects")) }\end{array}$ & 2072 \\
\hline$\# 1$ & $\begin{array}{l}\text { Search "Pulmonary Disease, Chronic Obstructive" [MeSH] OR "COPD" OR } \\
\text { "chronic obstructive pulmonary disease" }\end{array}$ & 32912 \\
\hline
\end{tabular}




\section{Appendix 2}

Figure 5. Summary of key differences with long-acting bronchodilators, ICS, or ICS+LABA versus placebo or comparator in post-dose exercise measures: (A) exercise endurance time; (B) Borg dyspnoea score; (c) inspiratory capacity (IC); and (d) tidal volume (data are presented as mean treatment ? placebo differences \pm SE (solid line) or with $95 \% \mathrm{Cl}$ (dashed lines), where available; $\left.{ }^{*} p<0.05, * * p<0.01, * * * p<0.001\right)$. (A) +Change from baseline in limit of exercise tolerance was significantly greater for FOR+TIO than FOR+PBO $(124 \pm 27 \%$ vs $68 \pm 14 \% ; p<0.05)$. $¥$ Added to bronchodilator therapy. (B) tAs expected at end of exercise (i.e. when patients stopped exercising owing to symptom limitation) the level of symptoms was the same in the two treatment arms. ¥Added to bronchodilator therapy. (C) †Measured at end of exercise, not isotime. ¥Added to bronchodilator therapy. (D) †Added to bronchodilator therapy. ACL=aclidinium; BUD=budesonide, FOR=formoterol, FP=fluticasone propionate, ICS=inhaled corticosteroid, IND=indacaterol, LABA=long-acting $\beta$-agonist, SLM=salmeterol, $\mathrm{PBO}=$ placebo, TIO=tiotropium

\section{A}

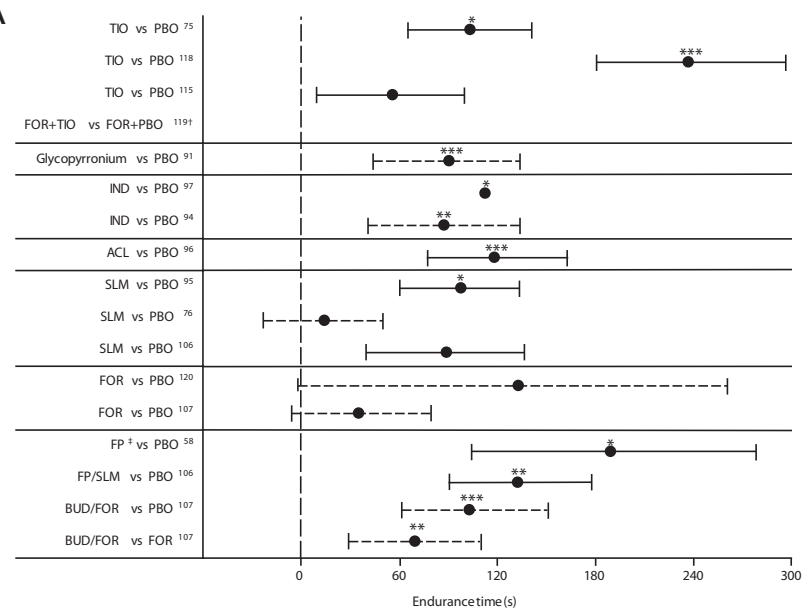

B

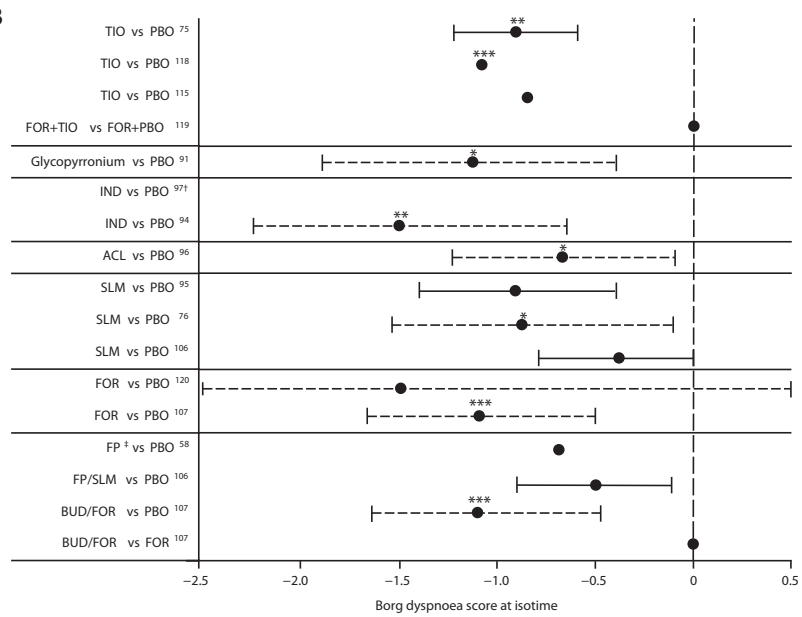

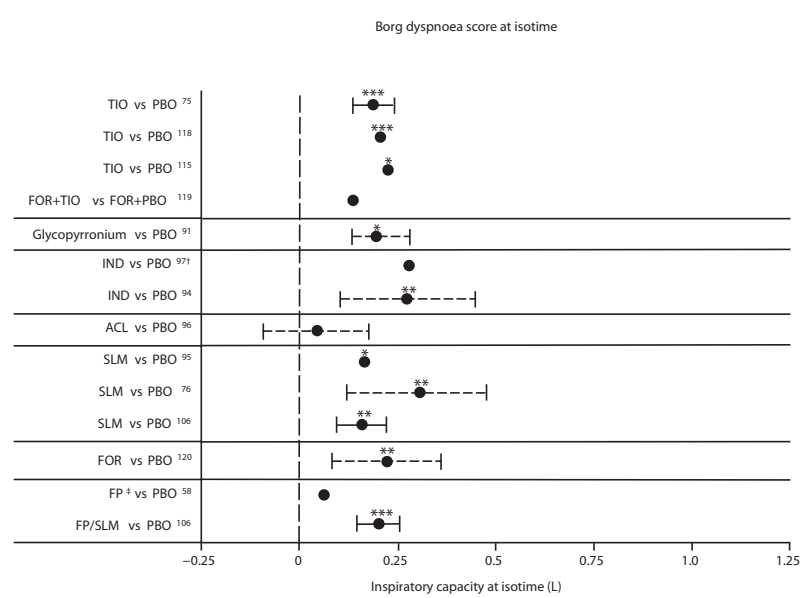

D

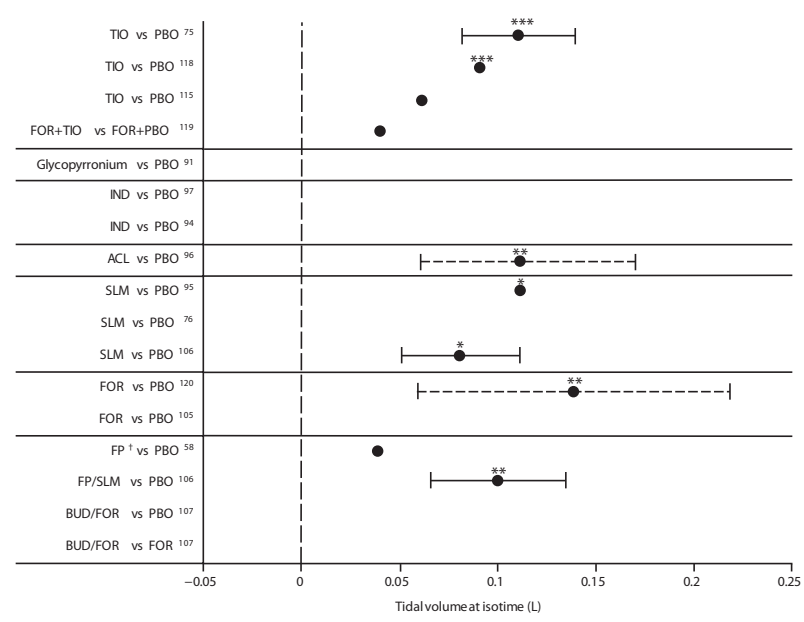

